\title{
The Electrical Response of the Isolated Fish Barbel
}

\author{
Eizo Yamashita \\ Department of Physiology (Prof. S. Tsukahara), \\ Fukushima Medical College, Fukushima
}

\begin{abstract}
Single unit discharges were recorded from the dissected nerves of the loach barbel in response to mechanical stimulation. The responses were divided into two types, one was phasic response and the other was a group of after-discharge. Among the fibers producing phasic response to stimuli the thin fibers had lower threshold in comparison with the thick fibers. Under the repetitive stimulation the barbel nerve could follow up to $70 / \mathrm{sec}$ at temperature of $25^{\circ} \mathrm{C}$, and discharge failed to follow at $180 / \mathrm{sec}$. A loach is likely to substitute the function of its barbels partly for that of its lateral-line.
\end{abstract}

Some fresh water fishes, for examples, the catfish, carp and loach have barbels. Their functions were investigated electrophysiologically by several workers. The taste response of barbels of these fishes were studied by Tateda, ${ }^{1}$ Konishi and Zottermann, ${ }^{2}$ Kumagai et al. ${ }^{3}$ These barbels also well respond to mechanical stimulation. It is known that the loach, which has five pairs of barbel, lives in muddy water and often gets into mud. Since visual sensation is useless in the mud the touch sensation of the barbel possibly much contributes to the behavior of the fish. In this paper the response to mechanical stimulation of the loach barbel was investigated.

\section{METHODS}

The isolated barbels of a loach were used. After decapitation the nerves innervating the barbels were removed from the surrounding tissues (Fig. 1) and were cut. Fig. 2 illustrates an arrangement of the preparation. A piece of perioral part of the fish containing barbels with nerves intact was isolated from the fish and was mounted on a small lucite holder $(\mathrm{H})$, and they were dipped into Ringer's solution covered with mineral oil. One of the nerve bundles from the barbel was dissected with steel needles until several fibers $(\mathrm{N})$ or occasionally single fiber were left intact. They were lifted and placed on a silver wire recording electrode $\left(\mathrm{E}_{1}\right)$ in the mineral oil layer. A similar silver wire was placed as an indifferent electrode $\left(\mathrm{E}_{2}\right)$ on the base of barbel. Mechanical stimulus to the barbel was given through a small glass tube (GT) cemented to the vibrating membrane of a magnetic speaker

Received for publication, November $15,1965$. 


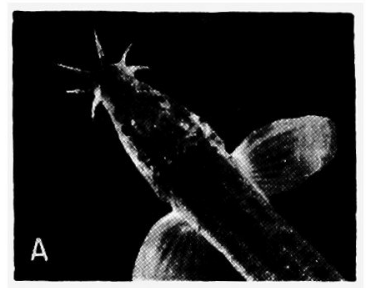

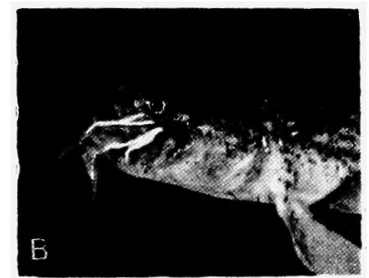

Fig, 1

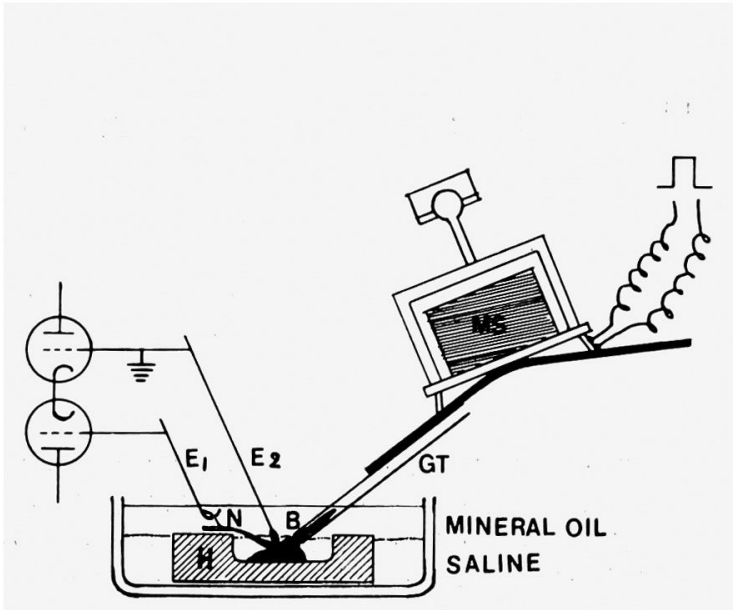

Fig. 2

Fig. 1: A: The head of a loach. B: The exposed nerves coming from the barbels.

Fig. 2. Arrangement of the preparation and experimental setup. Explanation in text.

(MS), which was activated by a pulse generator. Spikes from the nerve were amplified through an RC-coupled amplifier and displayed on a cathode ray oscilloscope. All the experiments were carried out at room temperatures of $15-25^{\circ} \mathrm{C}$.

\section{RESULTS}

\section{Various types of responses}

Spontaneous discharges were frequently obtained from the dissected afferent nerve fibers supplying the barbels, although many fibers without spontaneous discharge were observed which responded to mechanical stimuli applied to the barbels. Among the fibers producing the spontaneous discharges some responded to the stimuli, but others did not.

Some examples of spontaneous discharges are given in Figs. 3 and 4 . On the successive traces in the left column of Fig. $3 \mathrm{~A}$ a few spontaneous discharges are observed, while from the traces in the right column some change of spontaneous discharge is detectable after stimulation. In this experiment small unit spikes with the latency of about $15 \mathrm{msec}$ were evoked by the stimuli and were followed by groups of spikes much increased in number. In contrast to such a long lasting after-discharge there occurred a phasic response in other cases. An example is illustrated in Fig. 3B, in which the fiber generated a single spike in response to the stimulus but produced no after-discharge. Fig. 4A illustrates another example of discharge pattern. In this experiment two discharge units were detectable. One of them responded to the stimulus without after-discharges, while in the other unit the discharge was much decreased for about 20 msec follow- 
A

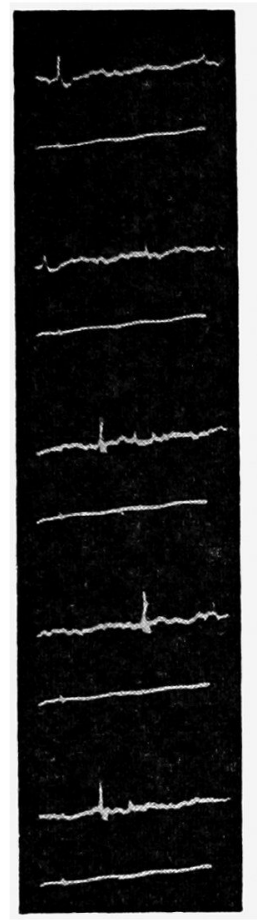

B

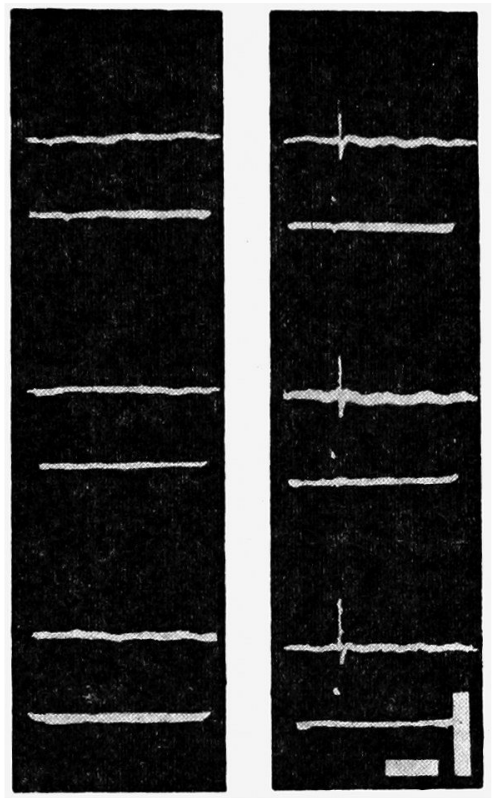

Fig. 3. The left columns of $\mathrm{A}$ and $\mathrm{B}$ indicate successive records obtained levery one second without stimuli, and right columns indicate the records of discharges in response to mechanical stimuli. A dot on the horizontal line below each record indicates stimulus moment. Vertical and horizontal bars indicate $200 \mu \mathrm{V}$ and $20 \mathrm{msec}$, respectively.

ing each stimulus until the burst of several spikes came again. There are two possibilities to explain the mechanism of this silent period. If this silent period is presumed to depend on inhibition, the burst following this period should be regarded as a postinhibitory burst. On the other hand, it is possible to suppose that the silent period is a long latency of the spike in response to the stimulus. In the experiments on the response to mechanical stimulation of frog's skin, Catton ${ }^{4}$ reported that the latency of a certain type spike was as long as $50 \mathrm{msec}$ when the stimulus was slow. Since such a type of response was seldom encountered in the barbel, it is difficult to decide which of the possibilities suggested above is more appropriate. Fig. 4B illustrates another example of discharge type. In this experiment the uppermost two traces of Fig. $4 \mathrm{~B}$ were obtained before application of stimulus, and the third trace was obtained during mechanical stimulation with light poking with a glass stylus. The fourth trace and lower ones show after-discharge. As shown on the bottom, two traces obtained about 50 seconds 
A

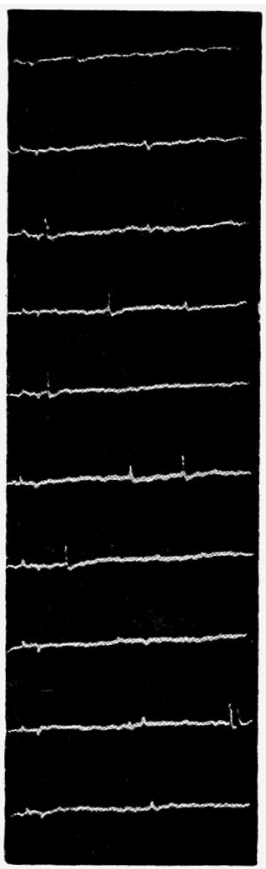

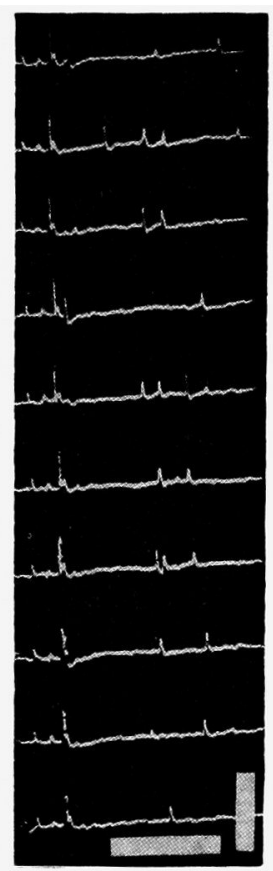

B

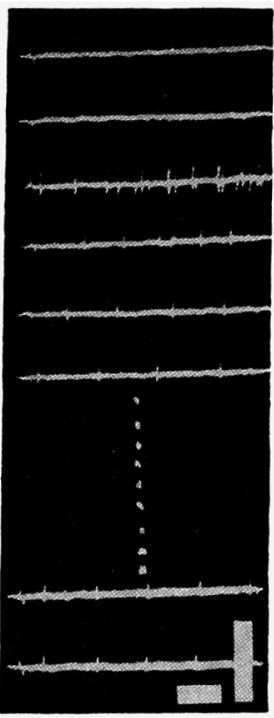

Fig. 4. A: After-discharge after stimulus and control without stimulus. B: After-discharge. Successive records are omitted until the last two records obtained 50 seconds later than stimulus. Vertical and horizontal bars indicate $200 \mu \mathrm{V}$ and $20 \mathrm{msec}$, respectively.

after the stimulus discharges were still observable at regular frequency, and they kept on for several minutes. The result suggests that the frequency change of spontaneous activity after stimulation depends partly on some hysteresis of the barbel tissue caused by sharp or strong mechanical stimulation. Katsuki et al. ${ }^{5}$ presented a similar conception on the difference between the tonic and phasic responses. Loewenstein ${ }^{6}$ found that the application of constant stretch to a frog's skin preparation altered the characteristics of the normally fast-adapting touch receptors so that they behaved in the same way as the more slowly adapting stretch receptors.

\section{The difference of threshold for mechanical stimulus}

A sharp and brief stimulus applied to the barbel by way of the stimulator produces only one spike in units of the phasic type when stimulus duration is mostly less than 2 msec. With an increased stimulus duration a single spike is produced at make and break of the stimulus. When the stimulus amplitude is low a spike is elicited more frequently at the off of the stimulus than at its on. 
But such specific distinction of on-unit and off-unit as reported by Hartline ${ }^{7}$ on the optic nerve is not found on the barbel. Repetitive spikes are not produced by the single mechanical stimulus owing probably to quick accomodation. Any potential changes corresponding to a generator potential have never been observed even if $0.5 \%$ solution of procaine was applied to the preparation to block the impulse initiation.

When the nerve bundle contains several units, the difference of thresholds is detectable. Fig. 5 illustrates an example of discharges under various stimulus

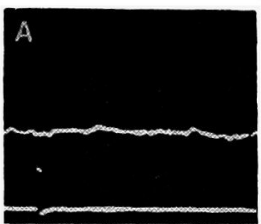

B

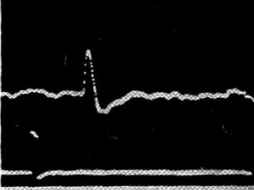

C

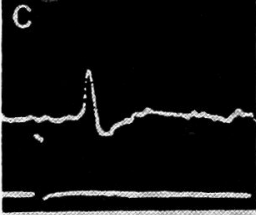

D

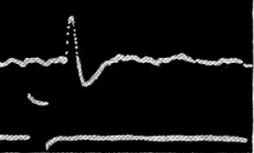

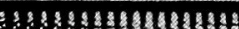
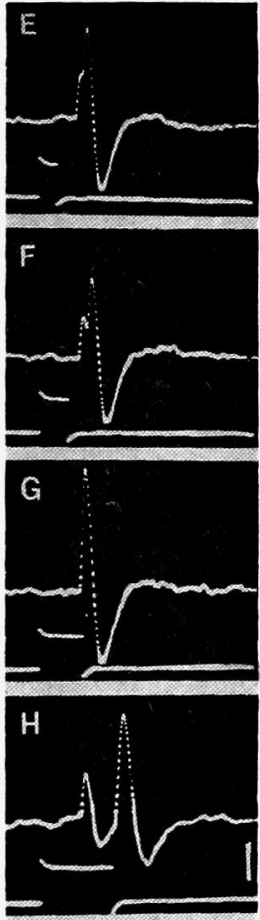

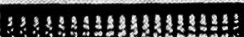

Fig. 5. Spikes in response to stimulus of gradually increasing duration. Vertical bar indicates $200 \mu \mathrm{V}$ and time mark $1,000 \mathrm{cps}$.

duration. In this experiment the stimulus duration was less than $2 \mathrm{msec}$ and a single small spike was produced (see B and D). In spite of increased stimulus amplitude the result was identical in $\mathrm{C}$. With gradual increase of the stimulus duration the second larger spike followed the initial smaller one $(\mathrm{E}-\mathrm{H})$. The amplitude of the second spike was apparently variable on account of probably algebraic summation of the first and second spike potentials. The similar results were obtainable many times on separate fibers in other experiments. Naturally 
large spikes are elicited from thick fibers and small spikes from thin ones. So, these results suggest that the thin fibers have lower threshold in comparison to the thick fibers. Such a phenomenon was observed by Katsuki et al. ${ }^{5}$ on the lateral-line nerve of an eel. The larger spikes were less frequently obtainable than the smaller ones, and the smaller spike sometimes was abolished with increased duration of stimulation. Besides these two kinds of spikes another several response patterns were observed, for example, some unit discharge was elicited by a light stroking of the barbel surface, but not by the stimulator.

\section{The effect of repetitive stimulation}

Under the repetitive stimulus with duration of 1-2 msec one spike is observable for each stimulus with a low repetitive rate. Fig. 6 shows an
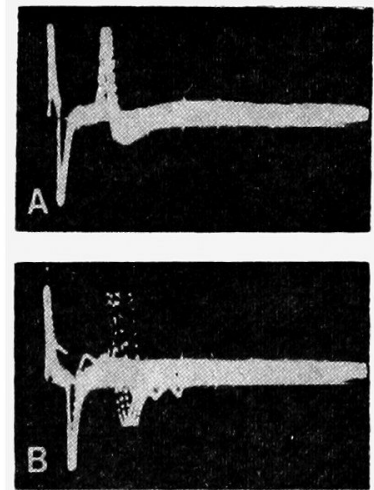

\section{DARAMAMALA}

Fig. 6. A indicates a record of ten superimposed traces talken under repetitive stimulus of $70 / \mathrm{sec}$ frequency and of $1 \mathrm{msec}$ duration. $\mathrm{B}$ is a similar record taken several ten seconds later than A. Time mark indicates 1,000 cps.

example of discharge in response to such a repetitive stimulation obtained at room temperature $25^{\circ} \mathrm{C}$. A record of about ten superimposed traces taken at a frequency of 70/sec with a stimulus duration of $1 \mathrm{msec}$ shows that the response still kept to appear to every stimulus. A train of stimulus applied over several ten seconds with the same frequency caused irregular prolongation of the latent time, and the falling out of spikes began to be observable (see B). It may be a sign of fatigue. And the falling out of spikes became gradually more manifest with higher frequency beyond 70/sec until spikes disappeared at 180/sec. The stimulus frequency at which the falling out of spikes begins is different from fiber to fiber and seems to be influenced by temperature. In most cases it is between $30 / \mathrm{sec}$ and $50 / \mathrm{sec}$ at $15^{\circ} \mathrm{C}$ and spikes are abolished before $100 /$ sec. The result shows that the barbel can respond to mechanical stimuli of considerably 
higher pitches. Parker and Hoagland ${ }^{8}$ observed that the lateral-line organs of fishes followed 20-70 cps. of vibratory stimulus. The loach barbel might follow the same frequency as the lateral-line.

\section{DISCUSSION}

Some nerve fibers contained in the same bundle coming from the barbel respond to the stimulus applied to the skin around the barbel. So, the touch sensation of the barbel is not its special function but is common to the body surface skin. Nerve fibers entering the skin terminate in arborizations of fine naked exoplasmic filament, if the fine structure of nerve ending in loach's skin is histologically similar to that in frog's skin. The microanatomy of the frog's subepidermal endings has been described by Whitear.' The barbel contains many nerve fibers of different thickness and of different thresholds. They might distinguish variable conditions of mechanical deformations with different response patterns. Although the physiological roles of spontaneous discharge have been still unknown, the results obtained in this experiment suggest that the frequency of spontaneous discharges is changeable after stimuli and under some conditions, and that spontaneous discharge could contribute to communicate some meaningful messages.

When the loach is swimming the flow or resistance of water may excite the touch sensation of the barbel and it may contribute to the control of the swimming of the fish. Since the lateral-line nerve is deeply embedded in muscle tissue of the loach, it is reasonable to consider that in the loach some parts of the lateral-line function are replaced by the function of barbels.

\section{References}

1) Tateda, H. The taste response of the isolated barbel of the catfish. Comp. Biochem. Physiol., 1964, 11, 367-378.

2) Konishi, J. \& Zotterman, Y. Taste functions in the carp. An electrophysiological study on gustatory fibers. Acta physiol. scand., 1961, 52, 150-161.

3) Kumagai, T., Nodai, H., Wada, M. \& Ogata, D. Über den Aktionspotential der Geschmacksnervenfasern von Fischen. Z. v. Kyushu Zahnarztliche Hochschule, $1952,6,1-4$.

4) Catton, W.T. Some properties of frog skin mechanoreceptors. J. Physiol. (Lond), 1958, 141, 305-322.

5) Katsuki, Y., Yoshino, S. \& Chen, J. Aotion current of the single lateral-line nerve fiber of fish. II. On the discharge due to stimulation. Jap. J. Physiol., $1950,1,179-194$.

6) Loewenstein, W.R. Excitation and changes in adaptation by stretch of mechanoreceptors. J. Physiol. (Lond.), 1956, 133, 588-602.

7) Hartline, H.K. The response of single optic nerve fibers of the vertebrate eye to illumination of the retina. Amer. J. Physiol., 1938, 121, 400 415.

8) Hoagland, H. Pacemakers in relation to aspects of behavior. Macmillan Co., London 1935 . 
9) Whitear, M. Dermal nerve endings in Rana and Bufo. Quart. J. micr. Sci., $1955,96,343-349$. 